\title{
A Review of the Effective Techniques of Compression in Medical Image Processing
}

\author{
Suma \\ Research Scholar, \\ Vidya Vikas Institute of Engineering \& Technology \\ Mysore, India
}

\author{
V Sridhar, Ph.D \\ PES College of Engineering \\ Mandya, India
}

\begin{abstract}
Medical image data (Ultrasonography, Computed Tomography, Magnetic Resonance Imaging etc.) consumes maximum storage and utilize maximum bandwidth for transmission that often results in degradation of image quality. Due to these inherent issues in such type of images, compression is the only applicable technique explored in the due course of prior research work. Currently, there exists abundant research work on medical image compression considering lossy and lossless types, but the need of medical images to be compressed efficiently with optimal compression ratio is yet a question mark. This paper will perform an investigation of various techniques explored and discusses some of the efficient techniques explicitly among all the prior work. While reviewing the prior literatures, it was explored that although medical image compression is an emerging need, but it encounters higher dimensionality of challenges and complicatedness for catering the increasing demands of the medical science.
\end{abstract}

\section{Keywords}

Medical Image Processing, Compression Techniques, Lossy and Lossless Compression

\section{INTRODUCTION}

In the past decade, there has been a substantial progress in the area of medical science. The hospitals started findings a way to store all the medical and radiological image in their database for further medical records. However, the scheme of storing the massive number of medical images is not that easy one as it calls for an efficient storage and retrieval technique too [1][2]. Digital medical images have potential benefits in terms of durability, portability and versatility. However, problems involving storage space and network bandwidth requirements arise when large volumes of images are to be stored or transmitted, as is the case with medical images [2]. Currently, all the big hospitals have the facilities of telemedicines and robotic surgery. In telemedicine, it is important that image should be compressed and sent via resource constraint network, while in robotic surgery, high definition videos are required for streaming with zero tolerance error [3][4][5]. However, even with such advancement in medical science, there is a gap between the medical science and technologies available to support it with an anticipated goal. It is very important that while performing compression on the medical images, the effectiveness of resolution as well as perceptual quality be restored. It is also known that compression is also accompanied by certain loss of significant information if the data are massive and channel capacity is highly limited for transmission purpose. Another difficult in the area of medical image compression is that all there are various types of biomedical images (x-ray, CT, MRI etc) and compression ratio applicable for all these bio-medical images highly differs from each other. Therefore, it can be seen that medical image compression is an important research issue regarding the degree of compression and the preservance of the relevant information. The difficulty, however, in several applications lies on the fact that, while high CRs are desired, the applicability of the reconstructed images depends on whether some significant characteristics of the original images are preserved after the compression and decompression process has been completed [6,7].

To optimize the above requirement i.e. the high compression ratios and the preservance of the relevant information, the context based compression is one of the best choices to achieve the optimum compression ratios without any loss of useful information which is basically done by selecting different important regions of an image along with the background and then compression methodology is applied in these regions separately and not on the whole image. The low compression level is applied to the useful regions while the high compression is applied on the un-important regions and the background. During this process again different regions are combined along with the background after removal of the redundant data. As a result, very high CRs are achieved by this methodology without any appreciable loss of information and clarity of the image.

In the proposed research paper, a brief discussion about the domain of compression in medical imaging is done and then the study is extended for investigating the prior compression techniques deployed. Section 2 discusses about the fundamentals of medical images followed by categories of compression applied in medical images in Section 3. Brief discussion of the standards of medical image compressions are done in Section 4 accompanied by techniques used in medical image compression in prior literatures in Section 5. This section mainly discusses about some of the effective schemes introduced by prior researchers. Section 6 discusses about the performance of the compression methods followed by concluding remarks of the paper in Section 7.

\section{SIGNIFICANCE OF COMPRESSION}

The complexities involved in the processing of the medical images significantly differs from that of ordinary images. The ordinary natural scene images are taken from generic digital image capturing devices, while the medical images are captured from a sophisticated image capturing devices. Usually, the images are not really colored in medical samples as colored image may not carry the significant clinical attributes that are of prime importance from diagnosis viewpoint. Hence, the medical images are normally grayscale or some other specific format that bears the clinical significance of the disease. Hence, a normal image processing algorithms are not directly applicable in medical images and 
requires some sort of enhancement to fit for the extraction of the diagnosis origin of the medical images.

The normal compression algorithm that are directly applicable in natural scene image if applied to medical image directly may result in loss of significant clinical information. Not only this, the conventional compression algorithm may not efficient lead to compress the medical data along with retaining the significant clinical information borne by the medical image. Usually, such types of images are quite heavier as compared to the natural scene image and hence find higher degree of challenges for long term archival and retrieval process in common servers in hospitals. It was also observed that existing compression technology cannot attain a comprehensive minimization of the size of bio-medical images as well as significant minimization of bit rate for transmission purpose. The last few decade has witnessed various sorts of compression algorithms towards reducing the optimal size of the bio-medical image along with retention of significant clinical information of higher importance for physician. This paper discusses only the few significant compression techniques previously attempted for compression bio-medical images. Majority of the prior techniques are studied with respect to lossless as well as lossy compressiontechniques. Some studies has integretedly addressed both the issues (lossless/lossy compression) together.

It was also noticed that majority of the prior research attempts were towards the direction of the lossless approach as communities pertaining to pathology was quite hesitant to implement lossy technique due to legal and authority issues. However, such research impediment were narrowed down, once the research community started visualizing the potential attributed of lossy techniques. Reviewing the literatures, it was found that there are dual mechanism of performing lossless image compression techniques that includes i) decorrelational technique and ii) image coding technique. The issues of redundancies are minimized by adopting medical image decorrelational technique. In the area of medical image processing, the redundancies can be represented as following:

2.1 Spectral Redundancies: It is a type of image redundancies that exists between multiple different spectral bands of an image.

2.2 Spatial Redundancies: It is a type of image redundancies that exists between all the neighboring pixels.

2.3 Temporal Redundancies: It is a type of image redundancies that exists between any adjacent frames of a video (or image sequences).

However, the literatures shows adoption of various techniques to perform minimization of redundancies existing within the bio-medical images. Some of such techniques are hierarchical interpolation, differential pulse code modulation, multiplicative autoregression, and bit-plane encoding. Some of the significant image encoding standards evolved in past and still in practice are Lempel-Ziv encoding, Huffman Encoding, Arithmatic encoding, and run-length encoding [8].

One of the common features of majority of the medical images are that it has potential correlation among the neighboring pixels and therefore the probabilities of existing image redundancies are also quite high. Solution of such issues are identified by determining the minimal correlation depiction on the medical image. It is quite important to address the redundancies issues while dealing with medical image compression as if the image is not compressed, than such redundancies will occupy more channel capacity and unnecessary storage. Although there are much advancement in the data storage, much still the area of medical image processing, the challenges still exists and continues to provoke the research communities to solve it. As majority of the medical records are stored in sorts of image or video files, most of them are of high definition multimedia files and formats, striking more challenging scenario for performing compression technique. Table 1 exhibits such challenging scenario of multimedia files in terms of storage and channel capacity. Hence performing compression technique using conventional algorithm may result in loss of significant data or less compatible for storage and efficient bandwidthm requirements.

Table 1. Challenges of storage and bandwidth for multimedia files

\begin{tabular}{|l|l|l|}
\hline Multimedia Data & Size/Duration & $\begin{array}{l}\text { Transmission } \\
\text { Bandwidth }\end{array}$ \\
\hline Single Page & $12^{\prime \prime} \times 8.3^{\prime \prime}$ & $33-65 \mathrm{~Kb} /$ page \\
\hline Telephonic Audio & $11 \mathrm{Sec}$ & $64 \mathrm{~Kb} / \mathrm{sec}$ \\
\hline Grayscale Image & $512 \times 512$ & $2.1 \mathrm{Mb} /$ image \\
\hline Colored Image & $512 \times 512$ & $6.29 \mathrm{Mb} /$ image \\
\hline Medical Image & $2048 \times 1680$ & $41.3 \mathrm{Mb} /$ image \\
\hline Super HD Image & $2048 \times 2048$ & $100 \mathrm{Mb} /$ image \\
\hline Full-motion Video & $640 \times 480,1$ & $221 \mathrm{Mb} / \mathrm{sec}$ \\
& Min $(30 \mathrm{frames} / \mathrm{Sec})$ & \\
\hline
\end{tabular}

\section{CATEGORIES OF COMPRESSION}

This section discusses about the categories of the compression technique found in the majority of the standard research work found to be adopted.

- Lossless vs. Lossy compression: Both the schemes are carried out to perform compression. The lossless approach ensures that the output image (reconstructed) to be equivalent to the original input image after performing compression. Unfortunately, the scheme can only attain minimalcompression. Even in lossy approach, the output image possess certain level of degradation as compared to original input image. The prime reason for this is because the redundancies lying within the image is rejected by the compression technique. Fortunately, lossy technique can accomplish higher degress of compression along with bettern retention of perceptual quality compared to lossless schemes [9][10].

- Predictive vs. Transform Coding: In prediction approach, the image information available while performing coding is deployed for performing forecasting of the future values while coding the error. Normally, it adopts image or spatial domain that is quite non-trivial to implement and is instantly adapted to local properties of 
an image. One of such example can be cited as differential pulse code modulation [11] technique. Transform coding preliminary transforms the medical image from its spatial domain representation of multiple types of representation using image transforms and coefficients were extracted after that. The coefficients were also termed as transformed values. Transform coding provide higher dimensionlity of data compression as compared to predictive technique. Transform coding has a technical flaw as it is computational expensive process for carrying out medical image compression.

\section{MEDICAL IMAGE COMPRESSION STANDARDS}

This section briefly discusses about the prior standards of performing medical image compression that has been either adopted or presented by majority of the researcher in the past.

\section{A. JPEG Predictive Lossless Standard}

This is one of the most frequently adopted technique in the area of medical image processing [12][13]. It consists of a predictor that integrates the values nearly to 3 neighboring pixels (Say A, B, and C) to formulate a forecasting of the image. The computation is perform to subtract the original value of the image and the difference is coded using lossless image compression. For this purpose, either Huffman or Arithmatic entropy coding process is used. Table 1 shows the list of predictors which is used in this technique. One dimensional predictors can be represented by selectional value of 1,2 , and 3 , while two dimensional predictors are represented as Selection 4, 5, 6, and 7. The selection value 0 is used only for the purpose of differential coding in hierarchical mode. Any significant image precision of size 2-16 bits/image can be used by the encoder and can use any of the predictors except selection-value 0 . The image precision is managed by the decoders along with other predictors too. A compression of $2: 1$ is generated by the lossless techniques exclusively for the color image with reasonably sophisticated scenes.

Table 2. Predictors Estimation Table

\begin{tabular}{|l|l|}
\hline Selection-value & Forecasting \\
\hline 0 & No Forecasting \\
\hline 1 & $\mathrm{~A}$ \\
\hline 2 & $\mathrm{~B}$ \\
\hline 3 & $\mathrm{C}$ \\
\hline 4 & $\mathrm{~A}+\mathrm{B}-\mathrm{C}$ \\
\hline 5 & $\mathrm{~A}+(\mathrm{B}-\mathrm{C})>>1$ \\
\hline 6 & $\mathrm{~B}+(\mathrm{A}-\mathrm{C})>>1$ \\
\hline 7 & $(\mathrm{~A}+\mathrm{B})>>1$ \\
\hline
\end{tabular}

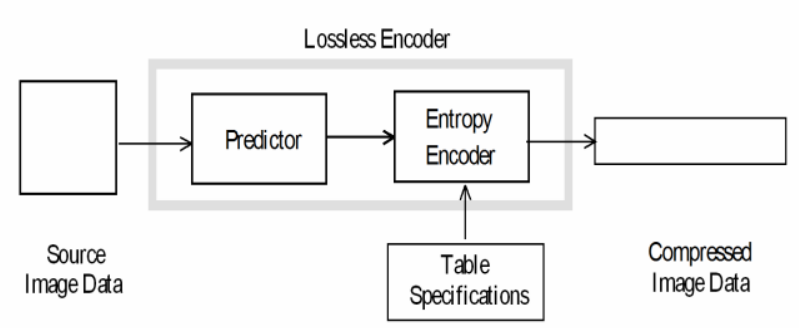

Fig 1: Mechanism of JPEG Predictive Lossless [12][13]

\section{B. The JPEG-LS Standard}

The JPEG-LS is the most recent technique adopted by many researcher for the purpose of compressing the medical image based on lossless approach. The design of the JPEG-LS is based on LOCO-I technique. LOCO refers to low complexity lossless compression for medical images [14][15] and is used for modelling image context mainly. The system uses context as the prime operator for performing sampling in the informal template for the purpose of performing coding of the current sample. The technique uses identification of probability distribution of forecasting error from the context.

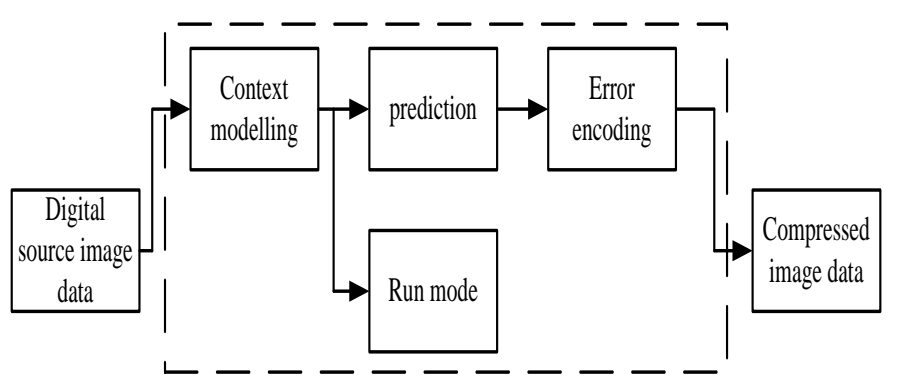

Fig 2: Illustration of JPEG-LS Standard

\section{Lossless JPEG 2000 Standard}

JPEG2000 is one of the most demanding technique of compression adopted by majority of the researcher in present era [16]. It is a type of an integrated lossless as well as lossy coding. The technique usually adopts wavelet transforms towards the input image which is subjected to scalar quantization. This act is closely associated with rate control mechanism.

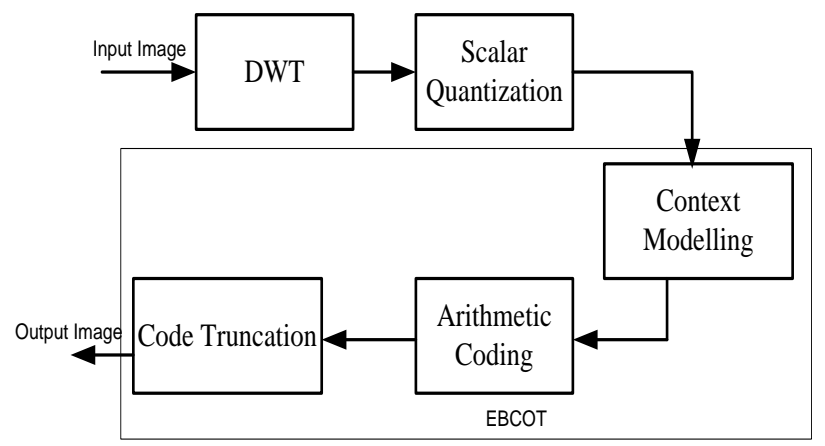

Fig 3: Illustration of JPEG2000 Standard

The system frequently adopts discrete wavelet transforms using mallat decomposition of the two channel filter banks in JPEG2000. As a part of code truncation function, EBCOT technique is applied [18][19], which is also called as post quantization. It is basically a bit-plane based image coding technique where the coefficients that are transformed are basically decomposed into bit-planes and are encoded using arithmetic coder. In some cases integer wavelet transform is also used in replacement of discrete wavelet transform and the technique skipps pre-quantization scheme.

\section{TECHNIQUES OF MEDICAL IMAGE COMPRESSION}

Majority of the hospitals who have their own diagnostic centers are required to store the radiological images for a comprehensive longer duration of time. Hence, along with storage issues, processing the radiological files to smaller size and performing transmission is one great challenges at present. Owing to this issues, medical image compression has gained 
its momemtum from research viewpoint. This section will discuss briefly some of the significant work in medical image compression in 2008-2012 considering the standard IEEE Transaction works.

\section{Advanced Video Coding Scheme}

Advanced video coding technique provides a better way to perform lossless image compression and is specifically designed for four dimensional medical image. The technique is based on advanced video encoding scheme e.g. H.264 as well as differential coding scheme for motion vectors. The scheme uses data redundancies in all the four dimensions thereby providing a better performance of compression. The redundancies within the volume are used by initially estimating the motion-compensated residual slices, which, in turn, are encoded using motion compensation across the temporal dimension. The quantitative experimental results on a large number of medical image datasets of varying modalities show significant improvements in compression ratio of up to three times that of current 2-D and 3-D state-of-the-art compression techniques, such as JPEG2000 and 3D-JPEG2000. The technique is highly flexible and is applicable in majority of the medical image compression techniques. The upcoming image formats and networking issues are handled to large extent by it.

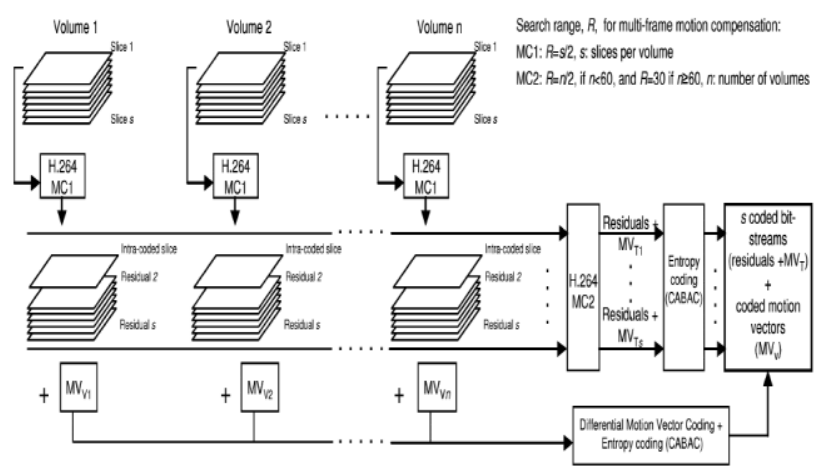

Fig 4: Lossless compression method for a 4-D medical image of $n$ volumes of $s$ slices [20]

2. Symmetry-Based Scalable Lossless Compression

Symmetry based scalable lossless image compression can be seen in study performed by Sanchez et al. [21]. The author have furnished a wavelet based technique for the purpose of compression for three dimensional medical image. Fig.5 shows the block diagram of the proposed scalable lossless compression method. Data decorrelation is performed by a $2 \mathrm{D}$ integer wavelet transform applied on slices within the medical image volume. The resulting sub-bands are then compressed independently by first employing a block-based intra-band prediction method to reduce their energy, followed by a modified version of the EBCOT algorithm to achieve resolution and quality scalability. The novelty of this intraband prediction method is in that it exploits anatomical symmetries within the structural data captured to predict the value of the wavelet coefficients on a block-by-block basis.

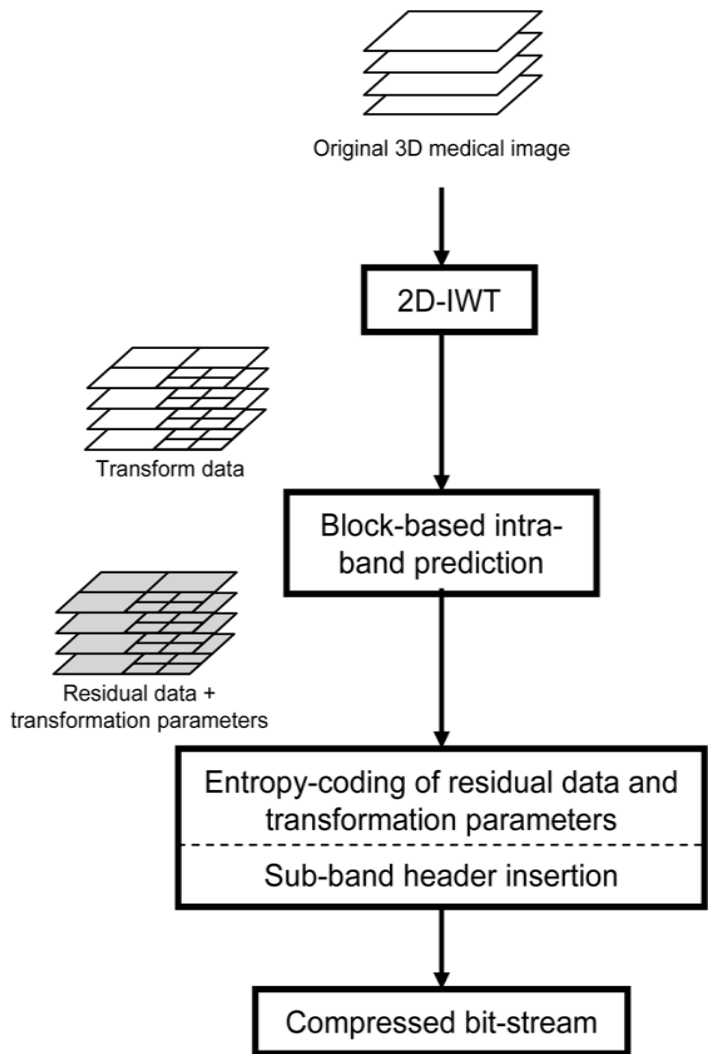

Fig 5: Symmetry based scalable lossless compression method-[21]

3. Compression Based on Motion Compensation and Customized Entropy Coding

Sanchez et al. [22] have briefed two major contributions that have acted as enhancement for compression of functional MRI (fMRI) data as a new multi-frame motion compensation process that employs 4-D search, variable-size block matching, and bidirectional prediction; and a new context-based adaptive binary arithmetic coder designed for lossless compression of the residual and motion vector data. The authors have validated the method on real fMRI sequences of various resolutions and compare the performance to two state-of-the-art methods: 4DJPEG2000 and H.264/AVC.

\section{Compression using JPEG-LS and Inter-Frame Coding}

Miaou et al. [23] have conferred a way that mixes the JPEGLS and an inter-frame encoding with motion vectors to boost the compression performance of deploying JPEG-LS alone. Here, an 8-bit grey-level image or an 8-bit color element of a color image is taken into account. The mechanism is exhibited in Fig.6. 


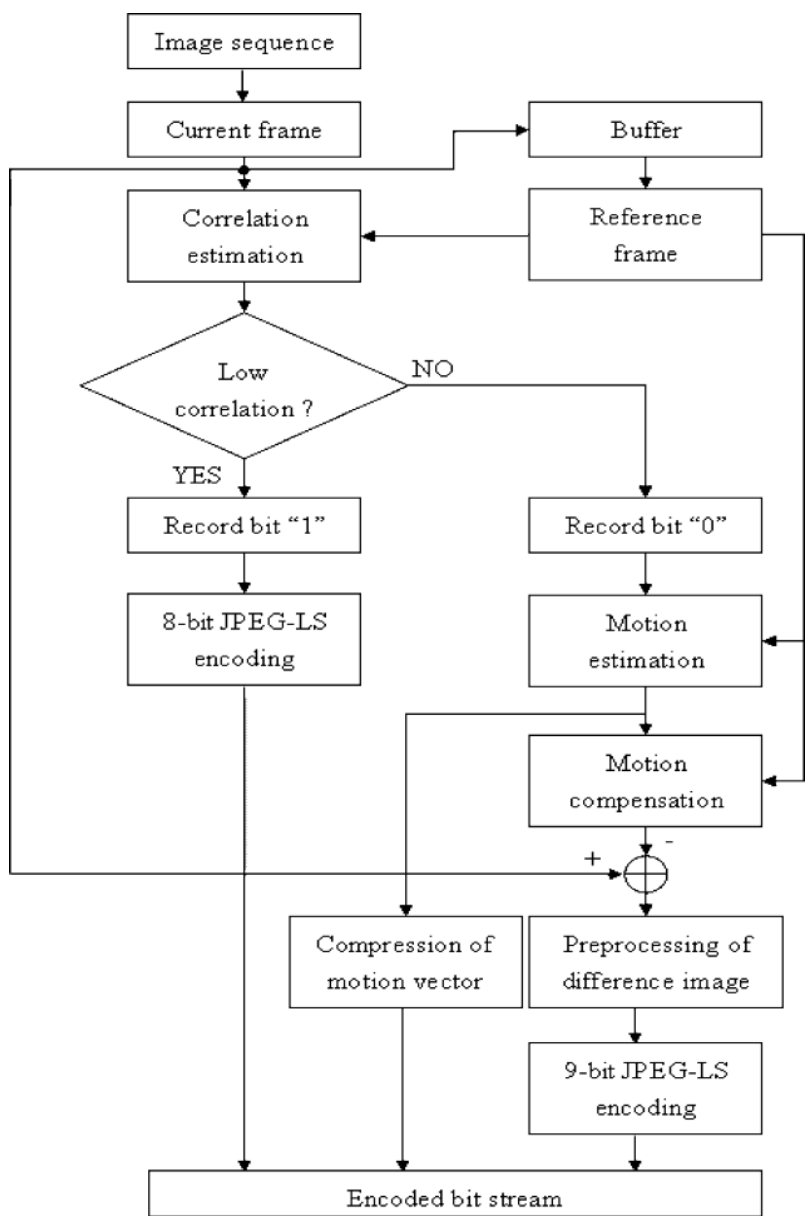

Fig 6: Compression using JPEG-LS [23]

Given a picture sequence, the primary input image is often compressed by 8-bit JPEG-LS encoding alone attributable to no frame of reference, then the second frame are going to be the present frame, and therefore the 1st frame becomes the frame of reference, and so on. First, the system has calculable the correlation between the present frame to be encoded and therefore the frame of reference within the buffer. If it's less than a correlation threshold, the system marks bit "1" as a flag and compress the present frame with 8-bit JPEG-LS writing. Otherwise, it marks bit " 0 " and perform motion estimation and motion compensation as in conventional video writing, followed by subtracting the reconstructed frame from the present frame to get a distinction image. The distinction image is then preprocessed and encoded by mistreatment 9-bit JPEGLS compression. The motion vector (MV) derived from motion estimation are going to be encoded. Finally, all the flag bits and therefore the encoded bits for one image frame area unit concatenated into single bit stream. This process cycle repeats for next image frame till the top of the image sequence. The decoder merely implements a reverse operation of encryption, together with commonplace 8-bit JPEG-LS secret writing or MV-based secret writing and 9-bit commonplace JPEG-LS secret writing. However, the performance of this compression technique differs from radiological images. For an example, theis method ensures around $27 \%$ compression gain for endoscopic images while it ensures around $86 \%$ compression gains for MRI images.
5. Compression with Optimized Volume of Interest Coding This techniques uses volume of interest coding exclusively designed for telemedicine applications [24]. The approach considers using three dimensional medical image for performing compression using integer wavelt transform. The technique also uses an enhanced version of EBCOT using the correlation among the wavelet coefficient and produces a measureable bit stream. The bit-streams are sorted and optimization principle is applied to performing image encoding targeting at highest perceptual quality.

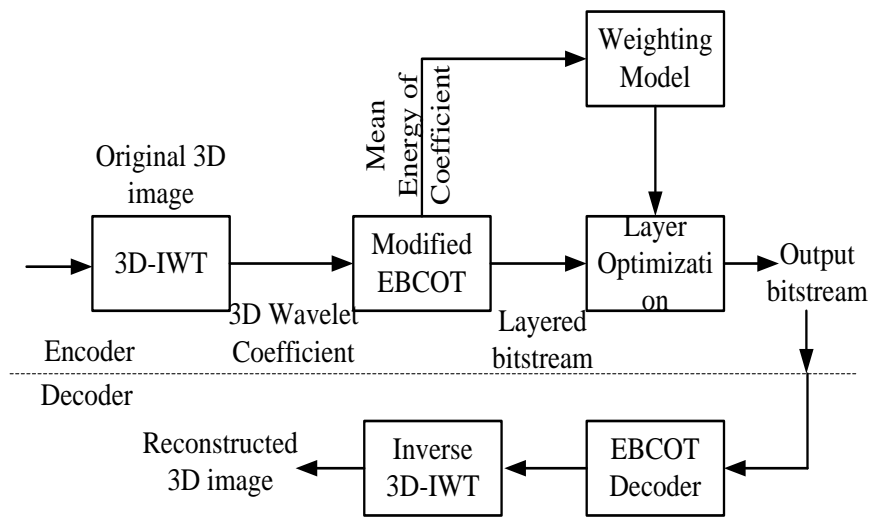

Fig 7: Compression with optimized VOI [24]

The technique has been assessed multiple number of time using real-time 8-12 bit medical images of three dimensional nature. The evaluation was done to show that the technique provides better resolution of various types of medical images. The outcome of the study shows that reconstructed image has higher degree of image resolution using potential features of JPEG2000 tested at numerous bit rates.

\section{Comparative Analysis of Computational Principles for JPEG2000}

Kim et al. [25] presented a technique for evaluating three image fidelity metrics of different computational principles-i) peak signal-to-noise ratio (PSNR), ii) high-dynamic range visual difference predictor (HDR-VDP), and iii) multi-scale structural similarity (MS-SSIM) - for the purpose of scaling the reliability of JPEG2000 standard compressed abdomen computed tomography images from a viewpoint of visually lossless compression.

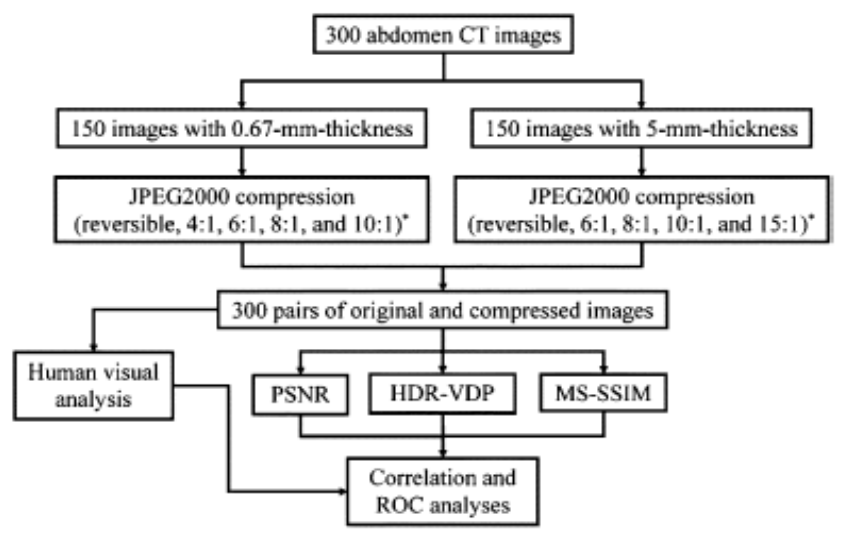

Fig 8: Study design-Source [25]

Fig.8 shows the mechanism of the [25], where the outcome of the study shows that the HDR-VDP outperforms the PSNR and 
MS-SSIM, and the MS-SSIM and PSNR are comparable in scaling the image reliability.

\section{Cross-Point Regions Approach}

This technique uses cross-point regions for performing lossless image compression evaluated over multiple bit planes. The technique is authored by Dang et al. [26] who have presented an algorithm for losslessly encoding and decoding images, exclusively for medical images, by enhancing the probability of bits on different bit planes of cross points that are neighbour points of grey levels $2^{\mathrm{n}}$.

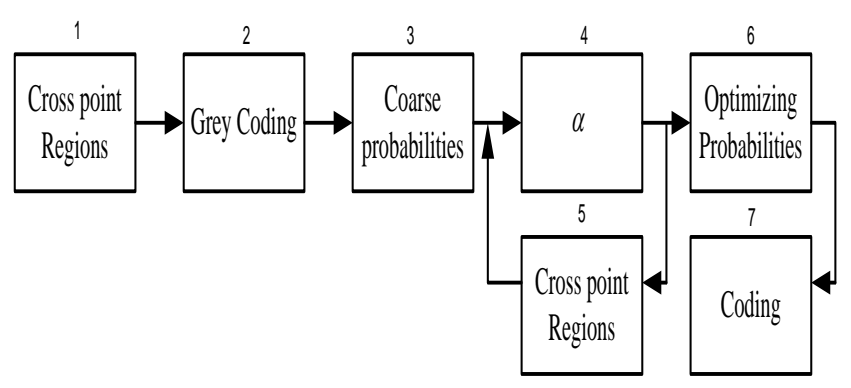

Fig 9: Cross-point region technique [26]

Fig. 9 presents a broad overview of the above scheme. The scheme has 7 phases. Phase 1 explores the region of cross point for enhancing the data bit probability. Phase 2 performs gray code transformation over the original data.. Phase-3 evaluates the probabilities of data bits in $\mathrm{k}^{\text {th }}$ order. Phase- 4 estimates the alpha value while phase 5 evaluates the cross point regions. This step will also explore exactly the regions where the probabilities of data bits need to be optimized by calculating factors a. Some cross-point regions satisfying step 1 but not satisfying step 5 will be cancelled. Step 6 enhances the likelihood of data bits. The process of encoding at step 7 (coding) uses Jones' algorithm it will give us the data, a compressed image.

\section{Hierarchical Oriented Predictions Approach}

Taquet et al. [27] have highlighted a hierarchical approach to resolution scalable lossless and near-lossless (NLS) compression that integrates the adaptability of DPCM schemes with new hierarchical oriented predictors to provide resolution scalability with better compression performances than the usual hierarchical interpolation predictor or the wavelet transform. A prediction level of the HOP scheme is performed in two steps. The first one (HStep) consists of predicting horizontally odd indexed pixels values with the aid of already known pixels: the proposed approach uses not only the even indexed ones but it can also take advantage of any previously predicted ones. Thus, one can obtain a horizontally sub- sampled image and the residual of the odd predicted pixels. The second step (VStep) is the mathematical transposition of HStep and acts on the lower resolution image.

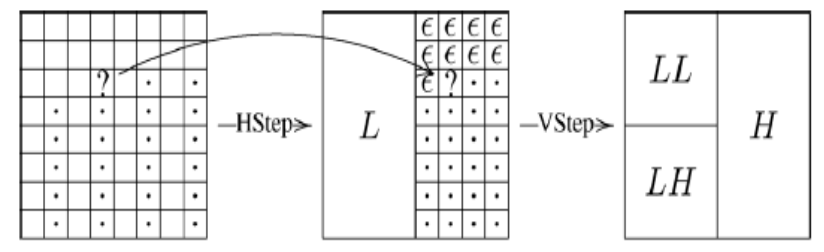
causal values
€ residual values
$\cdot$ non causal values
?? currently predicted

Fig 10: Hierarchical Oriented Prediction Approach [27]

\section{Automated Region-Based Hybrid Compression}

This technique uses an automated features for carrying out compression on an artificial region of a medical image. Evidence of such studies can be seen in the literature of Bairagi et al. [28]. The author has discussed an involuntary mechanism that performs hybrid compression on the medical images while ensuring minimal complexity on lossless images and highly scalable for DICOM images (DICOM images are most frequently used in medical studies and it stands for Digital Imaging and Communications in Medicine (DICOM) images

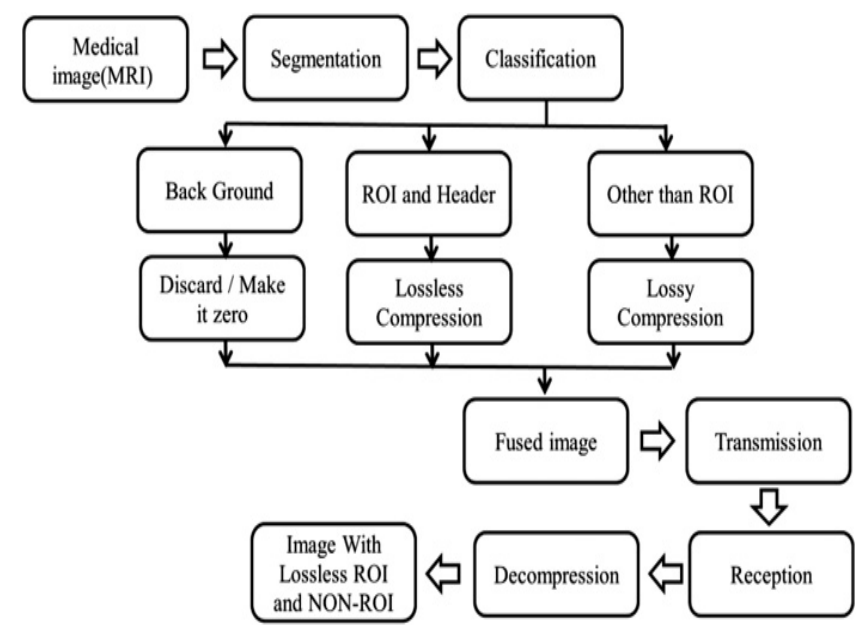

Fig11: Automated Region based hybrid compression [28]

The technique uses either computed tomography images or magnetic resonance images and posses three regions on the image e.g. i) region of interest, ii) non-region of interest, and iii) background. The mechanism of this technique is pictorially explained in Fig.11. Some more techniques of compression performance are shown in Table. 3

Table 3. Compression Performance of Medical Images [29]

\begin{tabular}{|l|l|l|l|l|l|l|l|l|}
\hline S. No & Property/Codec & JPEG & SPIHT & ASWDR & JPEG2K & EZW & SPECK & EBCOT \\
\hline 1 & $\begin{array}{l}\text { Targeted } \\
\text { Compression }\end{array}$ & No & Yes & Yes & Yes & Yes & Yes & Yes \\
\hline 2 & $\begin{array}{l}\text { Progressive } \\
\text { Transmission }\end{array}$ & Yes & Yes & Yes & Yes & Yes & Yes & - \\
\hline 3 & Low Memory & Yes & No & No & Yes & No & Yes & Yes \\
\hline 4 & $\begin{array}{l}\text { ROI/Context } \\
\text { Modelling }\end{array}$ & No & No & Yes & Yes & No & -- & Yes \\
\hline
\end{tabular}




\begin{tabular}{|c|c|c|c|c|c|c|c|c|}
\hline 5 & $\begin{array}{l}\text { Compressed } \\
\text { Operations }\end{array}$ & No & No & Yes & Yes & No & Yes & Yes \\
\hline 6 & Zero/Tree Concept & No & Yes & No & No & Yes & No & - \\
\hline 7 & SNR/PSNR & Poor & High & $\begin{array}{l}\text { Better than } \\
\text { SPIHT }\end{array}$ & Satisfactory & Good & $\begin{array}{l}\text { Better than } \\
\text { SPIHT }\end{array}$ & Good \\
\hline 8 & $\begin{array}{l}\text { Perceptual Image } \\
\text { Quality }\end{array}$ & Poor & $\begin{array}{l}\text { Better than } \\
\text { JPEG }\end{array}$ & $\begin{array}{l}\text { Better than } \\
\text { SPIHT }\end{array}$ & Satisfactory & Good & $\begin{array}{l}\text { Better than } \\
\text { SPIHT }\end{array}$ & Good \\
\hline 9 & $\begin{array}{l}\text { Compression } \\
\text { Ratios }\end{array}$ & Poor & High & High & Good & Better & Higher & Better \\
\hline 10 & $\begin{array}{ll}\text { Mean } & \text { Square } \\
\text { Errors } & \end{array}$ & High & Low & Lower & Satisfactory & Low & Lower & Low \\
\hline 11 & $\begin{array}{l}\text { Correlation } \\
\text { Coefficients }\end{array}$ & Low & High & Higher & Satisfactory & Good & Higher & Good \\
\hline 12 & $\begin{array}{l}\text { Computational } \\
\text { Complexity }\end{array}$ & Low & High & More & Yes & High & Low & Yes \\
\hline
\end{tabular}

\section{OPEN ISSUES/RESEARCH GAP}

This manuscript has discussed couple of the prior techniques are found to be frequently adopted in compressing medical images. From the review, it can be seen that adoption of certain standards like JPEG lossless compression is quite high as well as lossy compression techniques too [30]. For the techniques that implements discrete wavelet transforms, the perceptual quality of the medical image is found to be considerably high. For various radiological images (angio images), the wavelet based compression technique is found to be in acceptable limit in 25:1, while certain more types of images pertaining to ultrasounds are found to be in acceptable limits of 35:1 compression ratio [31]. However, in the area of telemedicine, where the images are also required to be transmitted, it was found that the compression ratio maximizes upto $30-50 \%$ for same medical images. It was also found that $\mathrm{x}$-ray based medical images have considerable more compression ratio acceptability as compared to computed tomography based medical images. However, the lossy compression technique seen on majority of chest radiological images, although found in acceptable compression ratio, but they fail to achieve significant minimization in the identification capability [32]. The processes that considers magnetic resonance images was found to preserve significant diagnostic integrity by deploying multi-spectral image compression. Interestingly, the lossless image compression doesn't produce much legal issues as the original input image is reconstructured in well manner with compression ratio up to 4:1 only. It should be known that lossy compression ratio differs from one to another dataset [33]. Hence, it can be said that the existing compression technique are although applicable in some radiological images but doesn't find much wider scope in terms of attaining better compression ratio. This facts calls for much in-depth investigation for novel approaches. Also, it was seen that majority of the prior studies is concentrated on lossy compression using numbers of image modalities without considering the region of diagnostic significance. Hence, a research gap is found that efficient technique for compression by emphasizing the region of diagnostic importance and discarding the other regions surfaces up. Working toward such issues may retain the clinical importance of the data carried by the region of diagnostic significance and will be helpful in future even for telemedicine. Hence, it is suggested that regionof-interest (ROI) scheme should be adopted as one of the alternative measure to overcome the side effects of compression on medical images. Using ROI technique can ensure better compression ratio towards the medical images.

\section{CONCLUSION}

This paper illustrates some of the standard prior techniques that has been studied in past to overcome the issues of compressing medical images. After reviewing the prior studies, it can be said that performance of medical image compression is highly dependent on compression ratio as well as perceptible quality of a reconstructed image. Reconstructed image with better perceptual quality will retain the information of the clinical importance to higher degree and will aid the diagnostic to have better result. Although abundant research work has been carried out in the past for exploring an efficient solution for performing medical image compression, but few of the techniques are actually applicable in the real time due to following issues e.g. i) the compression being performed on the entire image without undersdtanding the region of diagnostic importance, ii) existing compression technique doesn't ensure substantial perceptible quality of an image with optimal bit rate, iii) alternate solution of standard compression techniques are yet to be seen, iv) supportability of existing compression techniques on telemedicines are still unexplored. Moreover, none of the techniques have actually spoken about any mathematical techniques that is quite faster, efficient and reliable at the same time to perform compression on massive bio-medical images. Hence, our future direction of the work will continue towards designing a model for compression medical image that ensures better perceptual quality using region of diagnostic significance and thereby explore the possibilities of coming out with a novel mathematical model to be incorporated for enhancing the compression technique to higher degree.

\section{REFERENCES}

[1] Koller,L.2011. The Evolution of Medical Imaging Technologies: Electric Meat and Physician's Shifting Gaze, EA-Journal. Vol.2, pp.3.

[2] Yanga,C-T, Chen, C-H, Yang, M-F .2010. Implementation of a medical image file accessing system in co-allocation data grids, Future Generation Computer Systems. Elsevier. Vol.26, pp. 1127-1140

[3] Feng, D., Cai, W., and Fulton, R .2002. Dynamic image data compression in the spatial and temporal domains: 
clinical issues and assessment. IEEE Transaction on Information Technology in Biomedicine.Vol.6, pp.262268 , Issue. 4

[4] Portoni, L., Combi, C., Pinciroli, F.2002. User-Oriented Views in Health Care Information Systems. IEEE Transactions on Biomedical Engineering. Vol. 49, No. 12

[5] Al-Shaykh, O.K., and Mersereau, R.M.1996. Lossy compression of noisy cardiac image sequences. IEEE Proc. of the Data Compression Conf., pp.43-52, Vol. 31

[6] De Bruijn F.J., Slump C.H..1996. On the separation of quantum noise for cardiac X-ray image compression. Proc. of the 18th Annual Int. Conf. of the IEEE on Bridging Disciplines for Biomedicine. EMBS. Vol. 3, pp.1226 - 1227

[7] Ramaswamy, A., and Mikhael, W. B.1996. A Mixed Transform Approach for Efficient Compression of Medical Images. IEEE Trans. on Medical Imaging. Vol. 15 , No. 3

[8] http://www.newmediarepublic.com/dvideo/compression/ adv05.html

[9] Said, A., Pearlman, W.A.1993. An Image Multiresolution Representation for Lossless and Lossy Compression. IEEE Transactions on Image Processing

[10] Liu, C.M.. Lossless Image Compression, Retreived from www.csie.nctu.edu.tw/ cmliu/Courses/Compression/cha p7.pdf

[11] Arsinte, R., Miron, C. On the efficiency of the differential pulse code modulation in image coding and compression and an implementation on a digital signal processor based system. Retreived from citeseerx.ist.psu.edu/viewdoc/download?doi=10.1.1.182. 4359\&rep=rep $1 \&$ type $=$ pdf

[12] Acharya, T., Tsai, P.S. 2005.JPEG2000 Standard for Image Compression: Concepts, Algorithms and VLSI Architectures. John Wiley \& Sons, 21-Jan-2005 Computers, pp. 296

[13] http://en.wikipedia.org/wiki/Lossless_JPEG

[14] Rane, S.D., and Sapiro, G. 2001. Evaluation of JPEG-LS, the New Lossless and Controlled-Lossy Still Image Compression Standard for Compression of HighResolution Elevation Data. IEEE Transactions on Geoscience and Remote Sensing, Vol. 39, No. 10

[15] Ghrare, S.E., Ali, M.A., Jumari, K., and Ismail, M.2009. An Efficient Low Complexity Lossless Coding Algorithm for Medical Images. American Journal of Applied Sciences. Vol. 6 (8), pp.1502-1508

[16] http://www.digitalpreservation.gov/formats/fdd/fdd0001 39.shtml

[17] Chowdhury, M.M.H., and Khatun, A..2012. Image Compression Using Discrete Wavelet Transform. IJCSI International Journal of Computer Science Issues. Vol. 9, Issue 4 , No. 1

[18] http://www.jpeg.org/.demo/FAQJpeg2k/EBCOTcoding.htm

[19] Taubman,D.2000. High Performance Scalable Image Compression with EBCOT. IEEE Transactions on Image Processing
[20] Sanchez, V., Nasiopoulos, P., Abugharbieh, R.2008. Efficient Lossless Compression of 4-D Medical Images Based on the Advanced Video Coding Scheme. IEEE Transactions On Information Technology In Biomedicine, Vol. 12, No. 4

[21] Sanchez, V., Nasiopoulos, P., Abugharbieh, R.2009. Symmetry-Based Scalable Lossless Compression of 3D Medical Image Data. IEEE Transactions On Medical Imaging. Vol. 28, No. 7

[22] Sanchez, V., Nasiopoulos, P., Abugharbieh, R.2009. Novel Lossless fMRI Image Compression Based on Motion Compensation and Customized Entropy Coding. IEEE Transactions on Information Technology in Biomedicine. Vol. 13, No. 4

[23] Shaou-Gang Miaou, Fu-Sheng Ke, and Shu-Ching Chen.2009. A Lossless Compression Method for Medical Image Sequences Using JPEG-LS and Interframe Coding. IEEE Transactions On Information Technology In Biomedicine. Vol. 13, No. 5

[24] Sanchez, V., Nasiopoulos, P., Abugharbieh, R..2010. 3-D Scalable Medical Image Compression With Optimized Volume of Interest Coding, IEEE Transactions on Medical Imaging. Vol. 29, No. 10

[25] Kim, K.J., Kim, B., Mantiuk, R., Richter, T., Lee, H., Kang, H-S, Seo,J., and Lee, K.H. 2010. A Comparison of Three Image Fidelity Metrics of Different Computational Principles for JPEG2000 Compressed Abdomen CT Images, IEEE transactions on medical imaging. Vol. 29, No. 8

[26] Dang, T.T., Nguyen, S.K., Vu, T.D., Higuchi, S.2009. Cross-point regions on multiple bit planes for lossless images compression. IET Image Processing

[27] Taquet, J., and Labit, C.2012. Hierarchical Oriented Predictions for Resolution Scalable Lossless and NearLossless Compression of CT and MRI Biomedical Images. IEEE Transactions on Image Processing. Vol. 21 , No. 5

[28] Bairagi, V.K. Sapkal, A.M.2011. Automated regionbased hybrid compression for digital imaging and communications in medicine magnetic resonance imaging images for telemedicine applications. IET Science. Measurement and Technology

[29] Ansari, M.A., Anand, R.S., Recent trends in image compression and its application in telemedicine and Teleconsultation, national systems conference, NSC 2008, December 17-19, 2008

[30] Grgic, S., Grgic, M., Cihlar, B. Z.2001. Performance Analysis of Image Compression Using Wavelets. IEEE Transactions on Industrial Electronics, Vol. 48, No. 3

[31] Kurnaz, M.N., Dokur, Z., and Olmez, T.2002. Compression of MR andUltrasound Images by Using Wavelet Transform. Proc. of the 2nd Joint EMBS/BMES IEEE Conf., Houston, pp. 1021-1022

[32] Wong, S.1995. Radiologic Image Compression-A Review, IEEE Trans. on Medical Imaging. Vol. 83, No. 2

[33] LeeD.T.2005.JPEG.2000: Retrospective and New Developments, Proceedings of the IEEE. Vol. 93, No. 1 